\title{
Welcome and Opening Remarks
}

\author{
William D. Rhine ${ }^{1}$ and Lars Bode ${ }^{2}$
}

C ONSIDERABLE RESEARCH HAS demonstrated the positive effects of human milk on the growth and development of infants. Human milk has been shown to be particularly beneficial for preterm infants and other at-risk infants with unique nutritional and physiological needs. Current studies are continuing to explore not only the immediate benefits of human milk on infant development but also potential long-term benefits that may persist through adolescence and into adulthood. Researchers are also beginning to evaluate how maternal factors may influence breast milk composition and affect infant health and disease. Furthermore, research from an evolutionary, anthropologic, and/or bioethics perspective may help to better understand breastfeeding behavior and issues surrounding the use of donor human milk. Thus, scientists and clinicians from various disciplines are covering the full spectrum of human milk research.

The program for the 5th Annual International Conference on Human Milk Science and Innovation (ICHMSI), sponsored by Prolacta Bioscience, Inc., included presentations by leading international experts from a variety of fields. The scope of the conference covered a wide range of topics related to the scientific potential of human milk, its clinical applicability in different patient populations, and the history and ethics of human milk banking.

The 5th ICHMSI began with a keynote presentation on evolutionary, anthropologic, and biologic perspectives related to breastfeeding behavior and their potential impact on infant outcomes. The biology and science portion of the conference focused on the influence of human milk on the infant microbiome, including how variations in breast milk composition through diet or probiotic intervention may affect the gut mi- crobiome and immune responses. Human milk oligosaccharides and their potential role in reducing the risk of infections, such as necrotizing enterocolitis and rotavirus, were also discussed.

During the clinical portion of the conference, Dr. Adam Lewandowski was awarded the first Ruth A. Lawrence Investigator Award for Research in Human Milk Science for his research on the use of a human milk diet to minimize cardiovascular risk in preterm infants. This portion of the conference also included presentations on the impact of human milk on the mechanisms of transfusion-related acute gut injury and on brain development in preterm infants, and the use of human milk to treat infants with cystic fibrosis and children undergoing bone marrow transplantation. A comprehensive review on the history of human milk banking was also presented, as well as a discussion on the challenges of establishing human milk banks in the Muslim world.

Highlights from the 5th ICHMSI are captured in these proceedings. We hope continued discussions from this important meeting help to unite scientists and clinicians across disciplines to better understand the complexities and benefits of human milk and help to broaden the clinical application of human milk to different patient populations.

$$
\begin{array}{r}
\text { Address correspondence to: } \\
\text { William D. Rhine, MD } \\
\text { Stanford University School of Medicine } \\
750 \text { Welch Rd., Suite } 315 \\
\text { Palo Alto, CA } 94304
\end{array}
$$

E-mail:wrhine@stanford.edu

\footnotetext{
${ }^{1}$ Professor of Pediatrics (Neonatology), Stanford University School of Medicine, Palo Alto, California.

${ }^{2}$ Associate Professor and Larsson-Rosenquist Chair of Collaborative Human Milk Research, Department of Pediatrics and Larsson-Rosenquist Foundation Mother-Milk-Infant Center of Research Excellence (LRF MoMI CoRE), University of California, San Diego, La Jolla, California.
} 